\title{
The Alternative Pathway of Complement Activation in the Neonate
}

\author{
PETER A. FEINSTEIN AND STEPHEN R. KAPLAN ${ }^{(38)}$
}

The Sections of Medicine and Human Growth and Development of The Brown University Program in Medicine, and The Roger Williams General Hospital and The Women and Infants Hospital, Providence, Rhode Island, USA

\section{Extract}

C3PA (factor B) concentrations taken as an indication of alternate pathway development for neonates and adults were compared. The mean level for umbilical cord sera was $39 \pm 2 \%$, with a range of $19.5-77.5 \%$. The normal adult mean level was $74 \pm 4 \%$, with a range of $43-108 \%$. The difference between the two is highly significant $(P<0.001)$. The ratio of neonatal C3PA to adult C3PA is $0.52 \pm 0.10$. In only one case was the newborn level greater than the mean adult value. There is positive correlation, $r=0.18$, with gestational age, although it falls short of statistical significance $(P$ $>0.1$ ). There were no differences between the male and female neonates. C3PA titers were compared with $\mathrm{C} 3$ concentrations and so plotted. Although there was a positive correlation, $r=0.22$, it was not statistically significant $(P=0.1)$.

In an infant with gram-negative septicemia, the C3PA concentrations were much greater than the mean value found in normal cord sera. They were also greater than the mean value for normal adult C3PA titers, the multiple being $1.8 \cdot 2.5$. On first determination, after 2 days of normal to slightly elesated temperatures, a value of $132 \pm 6 \%$ was found. The second determination with a spike to $101.5^{\circ} \mathrm{F}$, and gave the highest of the three titers, $185 \pm 4 \%$. At the same time that the C3PA levels reached this peak, the fever dropped to normal. At the time of the last determination, the C3PA levels had returned to that of the original sample, $125 \pm 4 \%$.

This study demonstrates that the cord sera of the normal term neonate is deficient in $\mathrm{C} 3$ and C3PA when compared with adult controls. Neither C3 nor C3PA correlated with gestational age. C3PA levels increase steadily as C3 titers increase and comparable ratios to adult values indicate that the alternate pathway is probably maturing at the same rate as the classic pathway. The results in the septic infant may represent a response to an inflammatory condition (acute phase phenomena), a block in alternate pathway expression, or synthesis beyond increased C3PA catabolism.

\section{Speculation}

The development of the alternate pathway probably parallels the maturation of the organ systems responsible for its synthesis, although placental mechanisms (equilibration or production of an inhibitor substance) have not been excluded as a factor in the status of this system in the neonate. Investigation of the abnormal findings in an adequate sample of neonates with gram-negative is indicated to assess the role of the alternate pathway of complement activation in the pathogenesis and prognosis of this condition in the neonate.

The complement system is an important effector system in the pathogenesis of inflammation. An impressive array of biologic activities important to host defenses including immune adherence, opsonization, leukocyte chemotaxis, and virus neutralization result from activation of this system. The "classic" pathway of complement activation is initiated by an antigen-antibody complex activating the complex first complement component $(\mathrm{C} i)$, which in turn activates $\mathrm{C} 4$ and $\mathrm{C} 2$, and an enzymatic product is formed which cleaves 13 to its active state. As a result of the activation of C3 through C9, important biologically active products are also released, such as anaphylotoxins and chemotactic factors, and, ultimately, disruption of biomembranes resulting in cytolysis may occur (32).

In recent years it has been appreciated that another set of proteins, referred to as the "alternate" or "properdin" pathway, is capable of activating $\mathrm{C} 3(14,20,26,28)$, thus bypassing the initial antigen-antibody and $\mathrm{C} 1, \mathrm{C} 4$, and $\mathrm{C} 2$ reactions, but still producing the important biologic functions of the system $(12,17,24)$. Substances shown to be capable of initiating this pathway include endotoxin lipopolysaccharides $(11,14,16,21,28)$, aggregated myeloma proteins IgA $1, \lg A 2, \operatorname{IgG} 4$ (14), cobra venom factor ( 14 $27)$, and yeast cell wall zymosan $(6,29)$.

Despite the important role of the complement system in host defenses, the studies dealing with its development and function in fetal and neonatal life have been few. Except for the report by Stossel and coworkers (33), who correlated deficient opsonic activity of some cord serum with subnormal concentrations of $\mathrm{C} 3$ proactivator protein, the studies deal exclusively with the classic pathway components $(9,10,18,19,30)$. This study attempts to quantitate the maturation of the alternate pathway in the neonatal period.

\section{MATERIALS AND METHODS}

Adult sera from 17 healthy volunteers were obtained from the blood bank immediately after donation. The blood was allowed to clot and was then centrifuged. The resulting sera were stored at $4^{\circ}$ during the $4 \mathrm{hr}$ or less between centrifugation and time of freezing at $-91^{\circ}$ until used. Cord blood was obtained from 51 neonates at the time of delivery and processed in similar fashion. The three sera from infant $K$, a neonate suffering from gram-negative septicemia, were obtained by the heel prick method and stored as mentioned. One normal sample was initially designated as the reference serum for the protocol. This then served as the standard, to which the activity of all results were compared. Gestational age was obtained from the delivery room record at the Women and Infants Hospital at the time of delivery.

Alternate pathway activity was determined by measuring serum levels of one of its components, the $\mathrm{C} 3$ proactivator (C3PA or factor B) protein. C3PA levels were assayed by use of the radial immunodiffusion technique of Mancinni, as described by Ruddy et al. (31), in which only $8 \%$ or less variation is found between identical plates. The anti-human C3PA rabbit serum, lot no. $1548 \mathrm{~K}$ with an antibody concentration of $0.5 \mathrm{mg} / \mathrm{ml} \pm 30 \%$, was obtained from Behring Diagnostic Laboratories (34) and was stored at $4^{\circ}$. Plates were made of $3 \%$ Noble agar and buffer, with C3PA antiserum added in an amount resulting in a 1:40 dilution. The buffer was composed of $9 \mathrm{~g} \mathrm{Na-Veronal,} 65 \mathrm{mg} 0.1 \mathrm{~N} \mathrm{HCl}$, and $11 \mathrm{ml} 0.086 \mathrm{M}$ EDTA, diluted to 1 liter. Each plate contained $3 \mathrm{ml}$ agar-buffer-antibody mixture, and 20 wells were made, each having a diameter of $1.4 \mathrm{~mm}$. Each plate was duplicated. All plates contained three wells respectively, filled with the reference serum 


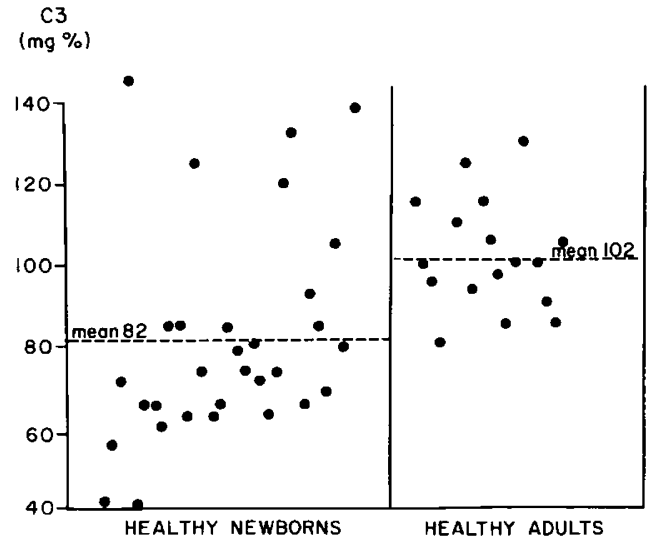

Fig. 1. C3 levels in healthy neonates compared with healthy adults.

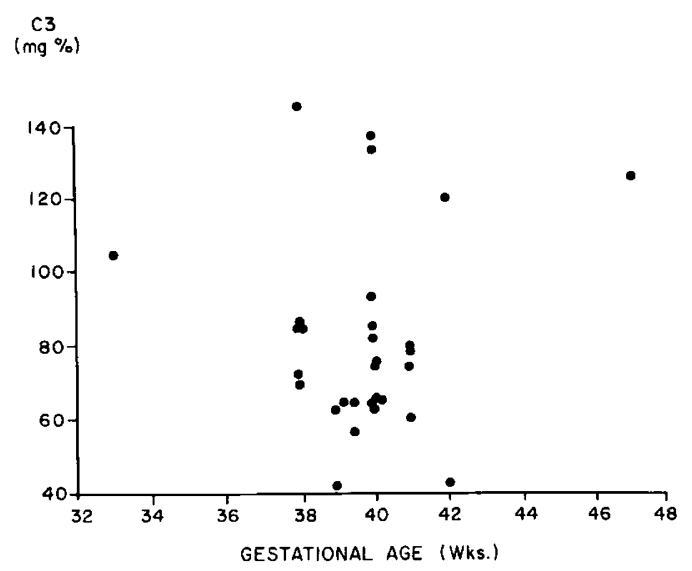

Fig. 2. relationship of $\mathrm{C} 3$ levels in neonates to gestational age.

diluted in $0.9 \%$ saline one-quarter, one-half, and full strength. Plates were read after $48 \mathrm{hr}$ of humid incubation at room temperature using a measuring magnifier (35). The diameter of the diffusion ring for each experimental sample was squared and compared with a concentration curve derived from the relationship between the serial dilutions of the reference sera and the squares of the diameter of each of their diffusion rings. Individual results are expressed as a percentage of reference serum activity. Results of each patient group are expressed as the mean reference serum activity for the group \pm SE. Statistical analysis utilized the Student $t$ test.

C3 levels were determined by the immunoplate human complement C3 method of Hyland Laboratories and quantitated using a log diameter method. Immunodiffusion of experimental sera in the agar-anti-C 3 protein-antibody medium was compared with three sera containing known $\mathrm{C} 3$ concentrations. A curve was composed relating diffusion data of the known sera to their $\mathrm{C} 3$ concentration. C3 level in the experimental group was derived from comparison to this curve. Results are expressed in milligrams per $100 \mathrm{ml}$ for each sample, and as the mean $\pm \mathrm{SE}$ for each group. Plates were read after $17.5 \mathrm{hr}$ of incubation at room temperature.

\section{RESULTS}

C3 levels of neonatal cord blood were significantly lower $(P<$ 0.005 ) than the $C 3$ levels found for adult sera (Fig. 1). The mean value for the cord sera was $82 \pm 4 \mathrm{mg} / 100 \mathrm{ml}$, with a range of $41-145 \mathrm{mg} / 100 \mathrm{ml}$. The mean C 3 value for adult sera was $102 \pm 3$ $\mathrm{mg} / 100 \mathrm{ml}$, with a range of $80-130 \mathrm{mg} / 100 \mathrm{ml}$. The ratio of neonatal to adult mean $\mathrm{C} 3$ concentrations was $0.80 \pm 0.9$, higher than the $0.54 \pm 0.14$ found to be the ratio of neonatal to maternal sera by others (9). C3 levels did not correlate with gestational age as obtained from the hospital record (Fig. 2), $r=0.06$.
C3PA concentrations, taken as an indication of alternate pathway development, are shown in Figure 3. The mean level for umbilical cord sera was $39 \pm 2 \%$, with a range of $19.5-77.5 \%$. The normal adult mean level was $74 \pm 4 \%$, with a range of $43-108 \%$. The difference between the two is highly significant $(P<0.001)$. The ratio of neonatal C3PA to adult C3PA is $0.52 \pm 0.10$. In only one case was the newborn level greater than the mean adult value. There is positive correlation, $r=0.18$, with gestational age (Fig. 4) although it fails to reach statistical significance $(P>0.1)$. The regression line is $\mathrm{C} 3 \mathrm{PA}=1.2$ (gestational age) -10 . There was no statistical difference between the male and female neonates. C3PA titers were compared with C3 concentrations for each sample and plotted in Figure 5. Although there was a positive correlation, $r=0.22$, it was not a statistically significant one $(P=$ $0.1)$. The regression line is $\mathrm{C} 3 \mathrm{PA}=0.09(\mathrm{C} 3)+2.7$.

The results of the three blood samples from infant $K$ are shown in Figure 6. Because there was no umbilical cord blood available, the three samples represent heel stick serum. On the second day of life, infant $K$ was diagnosed as having a gram-negative septicemia (criteria for diagnosis were temperature of $102^{\circ} \mathrm{F}$ and a lumbar puncture showing 24 white blood cells). Blood culture grew Escherichia coli sensitive to ampicillin and kanamycin 3 days later. On the third day of life, the infant was transfused with 25-30 cc whole adult blood. The samples were obtained after the transfusion, the first on day 5 (positive blood culture), the second on dav 11 , and the last on $d a v 18$. just before discharge.

The C3PA concentrations in the three samples are much greater than the mean value found in normal cord sera. They are also greater than the mean for normal adult C3PA titers, the multiple being 1.8-2.5. The first determination, on $d_{a} \cdot 5,2$ days after transfusion, and after 2 days of normal to slightly elevated temperature, gave a value of $132 \pm 6 \%$. The second, on dar $l l$, followed a 2-day period (davs 7-9) of elevated temperature with a spike to $101.5^{\circ} \mathrm{F}$, and gave the highest of the three titers, $185 \pm$

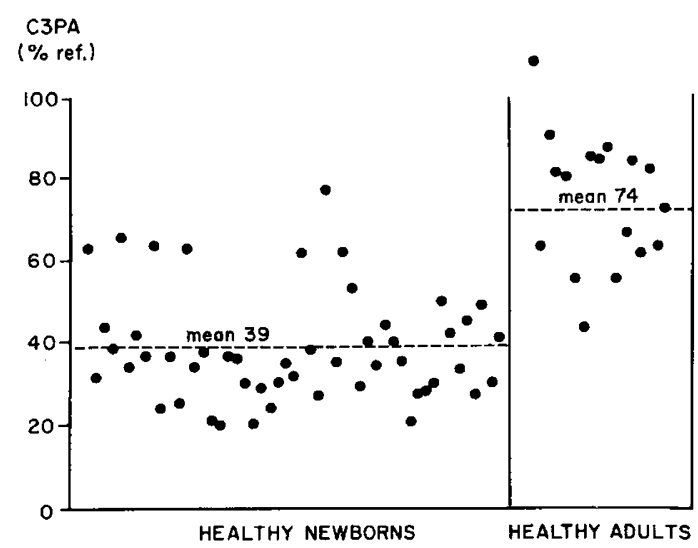

Fig. 3. C3PA levels in healthy neonates compared with healthy adults.

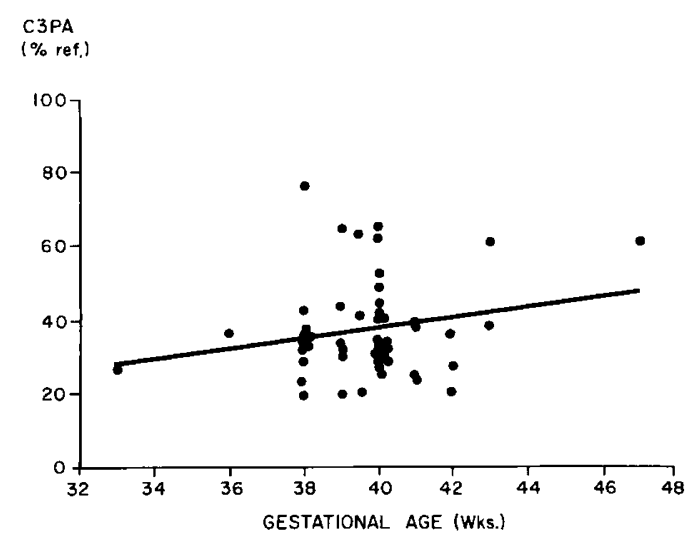

Fig. 4. relationship of C3PA levels in healthy neonates to gestational age. 


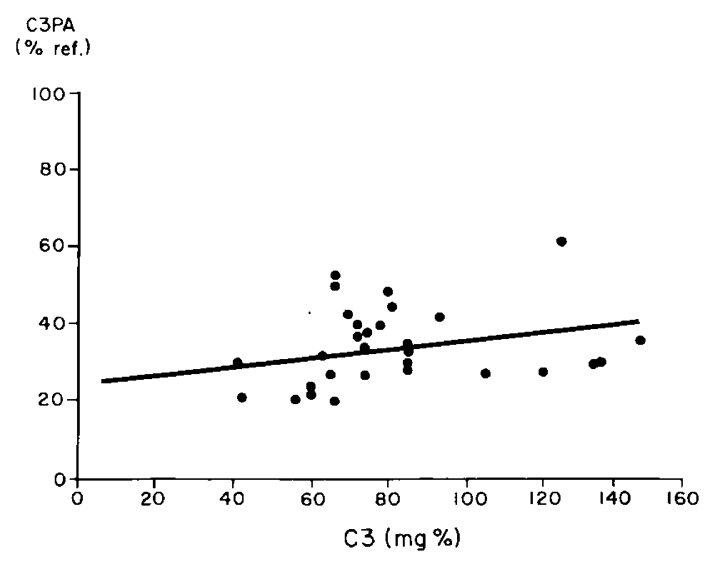

Fig. 5. relationship of $\mathrm{C} 3 \mathrm{PA}$ titers to $\mathrm{C} 3$ levels in healthy neonates.

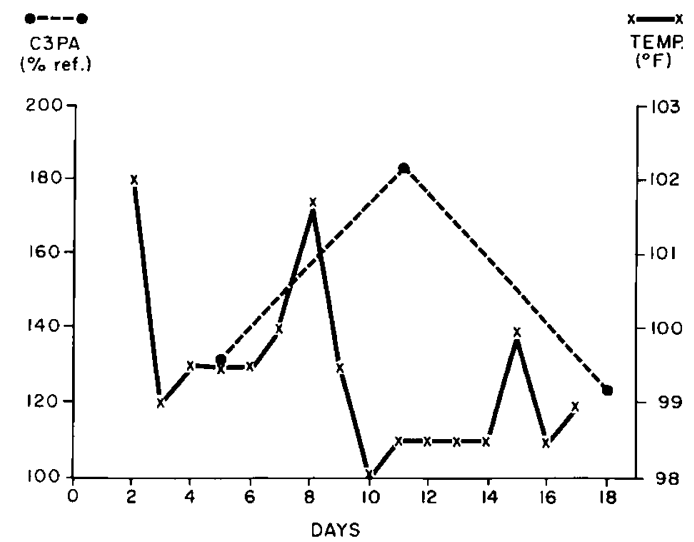

Fig. 6. relation of $\mathrm{C} 3 \mathrm{PA}$ to febrile course of gram-negative sepsis ( $E$. coli) in one neonate.

$4 \%$. At the same time that the C3PA levels reached this peak. the fever dropped to normal. At the time of the last determination, the C3PA levels had returned to that of the original sample $125 \pm 4 \%$ after a period of 7 days of normal temperature.

\section{DISCUSSION}

The $\mathrm{C} 3$ proactivator $(\mathrm{C} 3 \mathrm{PA})$ protein is one of several components of an alternate pathway of complement activation. The complete reaction sequence and function of this pathway remains a subject of investigation. C3PA is one of the terminal proteins of the alternative mechanism. Its activation results in the generation of split products with $\beta$ and $\gamma$ mobilities. The fragment with $\gamma$ mobility, C 3 activator (C $3 \mathrm{~A})$, exhibits C 3 convertase activity (14), causing depletion of the later classic pathway components (C3-C9), without activation of the earlier ones (C1, C4, C2). This study demonstrates that the cord sera of the normal term neonate is deficient in C3 and C3PA when compared with adult controls. In the case of the former, the findings of Fireman et al. (9) are confirmed. The C3PA ratio of infant cord sera to adult sera is 0.52 \pm 0.10 .

Fireman et al. (9) also found that $\mathrm{C}^{\prime} \mathrm{H}_{50}, \mathrm{C} 3, \mathrm{C} 4$, and $\mathrm{C} 5$ increased proportionally with gestation and reached adult concentrations within 36 months of age. In this study, neither C 3 nor C3PA behaved in this fashion when gestational age was taken from the delivery room record (Figs. 2 and 4). C3PA levels increase steadily as C3 titers increase (Fig. 5), and comparable ratios to adult values indicate that the alternate pathway is probably maturing at the same rate as the classic pathway.

It is possible that at a certain point in fetal life, the alternate pathway is "induced" to function fully because of a variety of factors contributing to the fetal immune system. Several studies have indicated that phylogenetically the complement system represents a more primitive biologic defense mechanism than the immunoglobulins $(1,7,13,18)$. For the fetus the alternate pathway of complement activation could be a more primitive mechanism than the classic pathway because the latter requires an antigenantibody complex for initiation $(25,32)$, whereas the former does not $(14,20,26,28)$.

The development of the alternate pathway may parallel the maturation of some organ system responsible for its synthesis: once this organ system is mature, its production of these components (C3PA) tapers until after birth. Kohler (18) has found that C3, C4, and C5 synthesis was uniformly present in multiple organ systems at 11 weeks of gestation and thereafter, but synthesis of the first complement component $(\mathrm{Clq})$ was limited almost exclusively to the spleen, began at 14 weeks, and was not uniformly present.

Placental transfer could account for the data, either by an equilibration effect or through elaboration of an inhibitor substance. No studies have as yet been done on C3PA regarding this possibility. For $\mathrm{C} 3$, results favor independent fetal synthesis. Fischel and Pearlman (10) studied complement components of paired mother-cord sera and concluded that transplacental equilibration was a strong possibility because of the constant ratios of the two. However. Propp and Alper (30) and Alper et al. (5) found allotypic differences in $\mathrm{C} 3$ between mother and fetus. Neither study found differences of an inhibitor substance.

The results for infant $K$ are important, as gram-negative infections are one of the more serious hazards of perinatal life. Endotoxic lipopolysaccharides are capable of activating the alternate pathway $(11,14,16,21,28)$, and many biologic aspects of endotoxic shock can be attributed to consumption of C3-C9 and their products $(12,15,17,24)$. McCabe et al. (22) have demonstrated that depletion of $\mathrm{C} 3$ in adults with gram-negative sepsis can be correlated with a poor prognosis. A similar study by Fearon (8) has shown that $\mathrm{C} 3 \mathrm{PA}$ levels were normal in uncomplicated adult cases of gram=negative sepsis, but were significantly depressed in patients whose course was complicated by shock. Alper has found increased susceptibility to infection in two patients, one with an abnormality of C3 $(2,3)$, the other with hypercatabolism of C3 (4). McCracken (23) found a significant relationship between C3 levels and opsonic activity for Staphylococcus aureus and Pseudomonas aeruginosa, increasing $\mathrm{C} 3$ levels corresponding to higher opsonic activity. A similar effect was found for $E$. coli infections (23). C3PA-depleted (by cobra venom) sera and untreated sera have been compared for ability to kill E. coli. In the former, $58.5 \%$ of the original $E$. coli survived treatment with the sera: in the latter, only $0.6 \%$ survived, making C3PA a requirement for normal bactericidal activity (14).

The results for infant $K$ may represent a response to an inflammatory condition (acute phase phenomena), a block in alternate pathway expression, or synthesis beyond increased C3PA catabolism. Investigation of our abnormal findings for this infant in an adequate sample of neonates with gram-negative sepsis, and a study of their C 3 and C3PA protein kinetics is indicated, to assess the rule of the alternate pathway of complement activation in the pathogenesis of gram-negative septicemia in the neonate.

\section{SUMMARY}

The alternate pathway of complement activation was studied in the neonate by measuring cord blood levels of one of its components, the $\mathrm{C} 3$ proactivator protein. When compared with adult serum titers of C3PA, neonates were found to be deficient. C3PA levels increased steadily as C 3 titers increased, and ratios comparable with adult values suggested that the alternate pathway is maturing at the same rate as the classic complement pathway. However, neither $\mathrm{C} 3$ nor $\mathrm{C} 3 \mathrm{PA}$ was found to correlate with gestational age.

In one infant with a gram-negative septicemia, the C3PA concentrations were much greater than the mean value found in normal cord sera, and $1.8 \cdot 2.5$ times greater than the mean value 
for normal adults. This finding may represent an acute phase phenomena, a block in alternate pathway expression, or synthesis of C3PA beyond catabolism.

\section{REFERENCES AND NOTES}

1. Adinolfi, M. B., Gardner, B., and Wood, C. B. Ontogenesis of two components of human complement: beta IE and beta IC-1A globulins. Nature, 219: 189 (1968).

2. Alper, C. A., Abramson, N., Johnston, R. B., Jandl, J. H., and Rosen, F. S. Increased susceptibility to infection associated with abnormalities of complement mediated functions and of the third component of complement. New Eng. J. Med., 282: 349 (1970)

3. Alper, C. A., Abramson, N., Johnston, R. B., Jandl, J. H., and Rosen, F. S. Studies in vivo and vitro on an abnormality in the metabolism of $\mathrm{C} 3$ in a patient with increased susceptibility to infection. J. Clin. Invest.. 49: 1975 (1970).

4. Alper, C. A., Block, K. J., and Rosen, F. S.: Increased susceptibility to infection in a patient with type II essential hypercatabolism of C3. New Eng. J. Med., 288: 601 (1973).

5. Alper, C. A., Boenisch, T., and Watson, L.: Genetic polymorphism in human glycine-rich beta glycoprotein. J. Exp. Med., 135: 68 (1972).

6. Blum. L.: Evidence for immunological specificity of the properdin system: Demonstration, isolation, and properties of a serum factor which interacts with zymosan and other polysaccharides at $0^{\circ} \mathrm{C}$. J. Immunol., 97: 61 (1964).

7. Day, N. D. B., Gewurz, H., Johannsen, R.. Finstad, J., and Good, R. A.: Complement and complement-like activity in lower vertebrates and invertebrates. J. Exp. Med., 132: 941 (1970).

8. Fearon, D. T., Ruddy, S., McCabe, W. R., and Schur, P. S.: Activation of the complement system by the properdin pathway in patients with gram negative bacteremia. J. Clin. Invest., 53: 23a (1974).

9. Fireman. P.. Zuchowski. D. A., and Taylor. P. M.: Development of the human complement system. J. Immunol., 103: 25 (1969).

10. Fishel, C. W., and Pearlman, D. S.: Complement components of paired mother-cord sera. Proc. Soc. Exp. Biol. Med., 107: 695 (1961)

II. Gewurz, H., Pickering, R. J., Synderman, R., Lichtenstein, L. M., Good. R. A., and Mergenhagen, S. E. Interactions of the complement system with endotoxic lipopolysaccharides in immunoglobulin-deficient sera. J. Exp. Med., 131: 817 (1970).

12. Gewurz. H., Shin, H. S., and Mergenhagen, S. E.: Interactions of the complement system with endotoxic lipopolysaccharides: Consumption of each of the six terminal complement components. J. Exp. Med., /28: 1049 (1968).

13. Gitlin. D., and Biasucci, A.: Development of $\gamma \mathrm{G}, \gamma \mathrm{A}, \gamma \mathrm{M}$, Blc/Bla, ClA esterase inhibitor, ceruloplasmin, transferrin, hemopexin, haptoglobin, fibrinogen. plasminogen, antitrypsin, orosomucoid, lipoprotein, macroglobulin and prealbumin in the human conceptus. J. Clin Invest., 48: 1433 (1969).

14. Gôtze, O., and Müller-Eberhard, H. J.: The C3-activator system: An alternate pathway of complement activation. J. Exp. Med., 134: 90s (1971).

15. Johnson, K. J., and Ward, P. A.: The requirement for serum complement in the detoxification of bacterial endotoxin. J. Immunol., 108: 611 (1972).

16. Kane. M. A.. May, J. E., and Frank, M. M.: Interactions of the classical and alternate complement pathway with endotoxin LPS: Effect on platelets and blood coagulation. J. Clin. Invest., 52: 370 (1973).

Copyright (c) 1975 International Pediatric Research Foundation, Inc.
17. Kitzmiller, J. L., Lueas, W. E., and Yelenosky, P. F.: The role of complement in leline endotoxin shock. Amer. J. Obstet. Gynecol., /I2: 414 (1972).

18. Kohler, P. F.: Maturation of the humatn complement system, onset time and sites of fetal Clq. C4. C3 and C5 synthesis. J. Clin. Invest., 52: 671 (1973).

19. Kohler, P. F.: Quantitative comparison of complement in mother and newborn Fed. Proc., 27: 491 (1968).

20. Lachmann, P. J., and Nichol, P.: Reaction mechanism of the alternate pathway of complement fixation. Lancet, 7801: 465 (1973).

21. Marcus, R. L., Shin, H. S., and Mayer, M. M.: An alternate complement pathway: C3-cleavage activity, not due to C4, $2 \mathrm{a}$, on endotoxic lipopolysaccharides after treatment with guinea pig serum: Relation to properdin. Proc. Nat. Acad. Sci. U.S.A., 68: 1351 (1971).

22. McCabe, W. R.: Serum complement levels in bacteremia due to gram negative organisms. New Eng. J. Med., 288: 21 (1973).

23. McCracken, G. H., and Eichenwald, H. F.: Leukocyte function and development of opsonic and complement activity in the neonate. Amer. J. Dis. Child., 12l: 120 (1971).

24. Mergenhagen. S. E., Snyderman, R., Gewurz. H., and Shin, H. S.: Significance of complement to the mechanism of action of endotoxin. Current Topics Microbiol. Immunol., 50: 37 (1969).

25. Müller-Eberhard. H. J.: Chemistry and reaction mechanisms of complement. Advances Immunol.. \&: 1 (1968).

26. Müller-Eberhard, H. J.. and Gotze. O.: C3 proactivator convertase and its mode of action. J. Exp. Med., 135: 1003 (1972)

27. Pickering, R. J. Wolfson, M. R. Good, R. A and Gerwurz, H. Passive hemolysis by serum of COVF: A new mechanism inducing membrane damage by complement. Proc. Nat. Acad. Sci. U.S.A., 62: 521 (1969).

28. Pillemer, L., Blum, L., Lepow, I. H., Ross, O. A.. Todd, E. W., and Wardlaw, A C.: The properdin system and immunity. I. Demonstration and isolation of : new serum protein, properdin, and its role in immune phenomena. Science, 120: 279 (1954).

29. Pillemer, L.: The nature of the properdin system and its interaction with polysaccharide complexes. Ann. N. Y. Acad. Sci., 66: 233 (1956).

30. Propp, R, A., and Alper, C. A.: C 3 synthesis in the human fetus and lack of placental passage. Science, 162: 672 (1968).

31. Ruddy, S., Carpenter, C. B., Müller-Eberhard, H. J., and Austen. K. F.: Complement component levels in hereditary angioneurotic edema and isolated C2 deficiency in man. In: P. A. Miescheu and P. Graber: Immunopathology. Fifth International Symposium, Mechanisms of Inflammation Induced by Immune Reactions p. 224 (Schwabe and Co., Basel, 1967).

32. Ruddy, S., Gigli, I., and Austen, K. F.: The complement system of man. New Eng. J. Med., 287: 489 (1972).

33. Stossel, T. P., Alper, C. A., and Rosen, F. S.: Opsonic activity in the newborn Role of properdin. Pediatrics, 52: 134 (1973).

34. Somerville, N.J.

35. Bausch \& Lomb. Rochester, N. Y

36. The authors wish to thank Dr. Leo Stern for his critical review in the preparation of the manuscript, and Miss Deborah Sweeney for her secretarial assistunce.

37. This research was supported in part by an Arthritis Clinical Research Center Grant from The Arthritis Foundation.

38. Requests for reprints should be addressed 10: S. R. Kaplan. M.D. The Department of Medicine. The Roger Williams General Hospital, 825 Chalkstone Ave.. Providence, R. I. 02908 (USA).

39. Accepted for publication June 25, 1975. 